Sharif University of Technology
Scientia Iranica
SCIENTIA
I RAN ICA
http://scientiairanica.sharif.edu

\title{
Wardrop's first principle: Extension for capacitated networks
}

\author{
H. Zokaei Aashtiani ${ }^{a}$, H. Poorzahedy ${ }^{a, *}$, and M. Nourinejad ${ }^{b}$ \\ a. Institute for Transportation Studies and Research (ITSR), Department of Civil Engineering, Sharif University of Technology \\ (SUT), Tehran, P.O. Box 14588 , Iran. \\ b. Department of Civil Engineering, University of Toronto, Toronto, Ontario M5S 1A4, Canada.
}

Received 4 March 2019; accepted 10 February 2020

\section{KEYWORDS \\ Equilibrium flow; \\ Wardrop principle; \\ Nash equilibrium; \\ Link capacity; \\ Transportation \\ network.}

\begin{abstract}
In transportation literature, User Equilibrium (UE) has been widely studied since early 1950's, many studies of which define equilibrium flow of traffic for uncapacitated networks based on Wardrop's first principle, implying also a Nash Equilibrium (NE). Although, in general, the two equilibria (UE and NE) are not explicitly the same, they are shown to be equivalent under special conditions for uncapacitated UE, when volumedelay functions are separable, continuous, non-decreasing and non-negative. A good deal of research is devoted to explain UE in capacitated networks based on Wardrop's first principle and the concept of generalized costs. However, UE for capacitated networks, even under the defined special conditions, is not equivalent to NE. This paper extends Wardrop's first principle to explain UE in capacitated networks, which, under the same special conditions of uncapacitated networks, would represent an NE as well. Moreover, a complementarity equilibrium model is proposed for UE, based on an extension of Wardrop's principle.
\end{abstract}

(C) 2021 Sharif University of Technology. All rights reserved.

\section{Introduction}

Any system has a capacity, and so do the links in a network. Two early concepts of link capacity are presented as: (a) The brick-wall capacity (e.g., the view taken by Highway Capacity Manual for road segments); (b) Deterring facility effect, as advocated by the FHWA Bureau of Public Roads (BPR) function (see, e.g., [1]), and used in BMW [2]).

The latter concept has been widely accepted in network problems, as it gives a friendly monotonic,

\footnotetext{
*. Corresponding author.

E-mail addresses: ashtiani@sharif.edu (H. Zokaei Aashtiani); porzahed@sharif.edu (H. Poorzahedy); mehdi.nourinejad@mail.utoronto.ca (M. Nourinejad)
}

doi: $10.24200 /$ sci. 2020.21736 convex and (high degree) differentiable function. However, this concept of link capacity, while producing reasonable link flows, has long been a source of concern in the computation of network performance measures, e.g. the total travel cost of the network: To deter the flow from entering a congested link, it offers high (usually unreal) user cost, which over-estimates the total travel cost (time) in the network. The former concept, however, may diminish this risk, but is computationally far unfriendly in practice.

In Nash Equilibrium (NE), no user may reduce self-travel time by unilaterally changing the path. The Wardrop's first principle for User Equilibrium (UE) states that the used paths travel times between an $\mathrm{O} / \mathrm{D}$ pair are less than, or equal to, the travel times of the other paths. These two equilibria are shown to be equivalent under special conditions for the uncapacitated UE, when volume-delay functions 
are separable, continuous, non-decreasing and nonnegative. Despite the wide application of Wardrop's first principle in many novel contexts, such as robust equilibrium [3], day-to-day equilibrium [4] and tradable credit schemes [5], very little is known about the implications of Wardrop's first principle in capacitated networks. Marcotte et al. [6] showed that defining UE for capacitated networks is a non-trivial task. They emphasized that Wardrop's first principle does not readily apply to it in its original form. Instead, they presented the following definition, which is a natural extension of Wardrop's first principle for equilibrium flow in capacitated networks.

Definition 1 [6]. "At equilibrium, the cost of a path with a positive residual capacity is larger, or equal to, the cost of any path carrying positive flow."

We present a schematic representation of Definition 1, using a simple example, and we use the results as a ground (motivation) for our study. Figure 1 illustrates a simple network with a single origin node 1 and two destination nodes 3 and 4 . Node 2 is a transition one. The rates of demand for $\mathrm{O} / \mathrm{D}$ pairs $(1,3)$ and $(1,4)$ are 1 and 2 , respectively. There are two alternative paths for each O/D pair, one directly from origin to destination and the other (alternative) indirectly from origin to destination through node 2 . We number these four paths 1 to 4 , respectively denoting direct and indirect paths from origin 1 to destination 3 , and indirect and direct paths from origin 1 to destination 4 .

The travel time (function) of each link is written on it; three links have constant travel times, and the travel time of the other links are functions of the respective flows $(x)$. The indirect paths to the two destinations share link $(1,2)$ with capacity of 1 unit of flow, and the other four links have unlimited capacities. Let $f_{k}$ be the flow in path $k=1$ to 4 . Then, the vector of flows, $f$, is feasible, if $f_{1}+f_{2}=1, f_{3}+f_{4}=2$ (conservation of flow), $f_{2}+f_{3} \leq 1$ (common link 2 capacity), and $f_{k} \geq 0, k=1$ to 4 (non-negativity of flow). These relations indicate:

$$
0 \leq f_{3} \leq 1-f_{2}=1-\left(1-f_{1}\right)=f_{1} \leq 1
$$

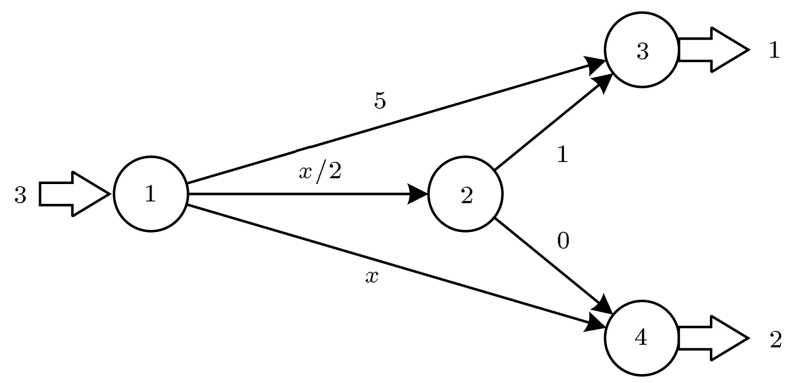

Figure 1. Motivating example network.
That is, $f$ is feasible, if $0 \leq f_{3} \leq f_{1} \leq 1$. This is shown in Figure 2 as the points inside the triangle $\mathrm{ABE}$. For the points on line $\mathrm{AB}$, that is where $0 \leq$ $f_{3}=f_{1} \leq 1$, the flow in the common link $(1,2)$, for the two paths 2 and 3 , reaches its capacity: $f_{2}+f_{3}=$ $\left(1-f_{1}\right)+f_{3}=1$. For the points on line $\mathrm{AB}$, the path travel times become:

$$
\begin{aligned}
& T_{1}=5, \\
& T_{2}=\frac{f_{2}+f_{3}}{2}+1=\frac{1}{2}+1=1.5, \\
& T_{3}=\frac{f_{2}+f_{3}}{2}+0=\frac{1}{2}+0=0.5, \\
& T_{4}=f_{4}=2-f_{3} \geq 1 .
\end{aligned}
$$

This means that for the points on line AB, for which $0 \leq f_{3}=f_{1} \leq 1$, we have $T_{1}>T_{2}$ and $T_{4}>T_{3}$, and that the common link $(1,2)$ of the paths 2 and 3 reaches its capacity. Hence, the flows corresponding to the points on line $\mathrm{AB}$ are in equilibrium, according to Definition 1. Moreover, note that for all of these points no flow of $\mathrm{O} / \mathrm{D}$ demand $(1,3)$ may be transferred from path 1 to path 2 (top path to middle path), and neither may any flow of $\mathrm{O} / \mathrm{D}$ demand $(1,4)$ be transferred from path 4 to path 3 (bottom path to middle path) because of the carrying capacity of link $(1,2)$. This means that these flows are in NE, but not in Wardrop's UE, since the travel times for paths with positive flow are not necessarily less than the travel times of the other paths.

It is also interesting to note that the total travel times in the network reduce from 6.5 at point $\mathrm{B}$ to 5.5 at point $\mathrm{A}$ (which is the only solution to BMW model) in Figure 2. This shows that link capacities in networks may adversely affect some users (here, $(1,3)$ $\mathrm{O} / \mathrm{D}$ demand) more than the others (here, $(1,4) \mathrm{O} / \mathrm{D}$ demand). By preventing the users of $\mathrm{O} / \mathrm{D}(1,4)$ from using link 2 (either partially or fully) in the example network, it is possible to raise equity and reduce

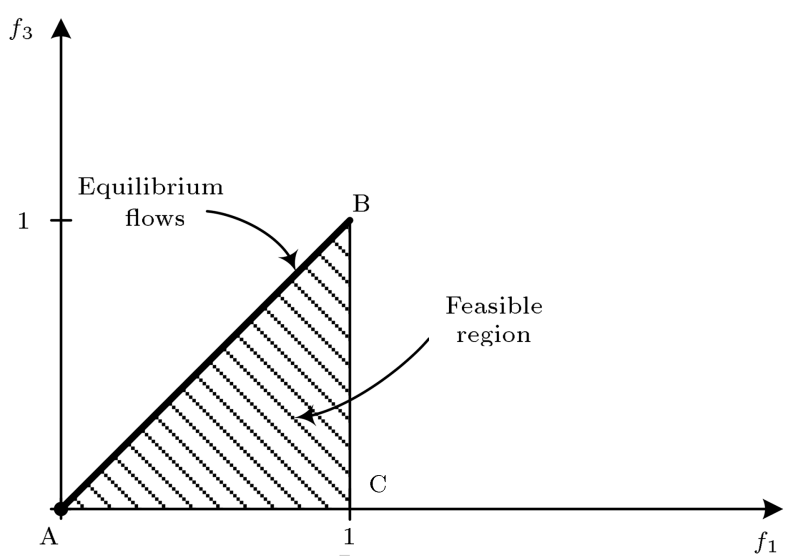

Figure 2. Equilibrium points (dark line) for the example network. 
the total cost of the network (increase efficiency). This may be done by pricing $(1,4)$ demand. The above observation may imply important managerial implications for the operators of the networks.

This paper intends to identify all such equilibrium points by the help of a complementarity equilibrium model. Although it is easy to identify NE for simple problems, the task of finding this equilibrium for larger problems cannot be accomplished easily, thus emphasizing a need for mathematical models that represent the comprehensive set of equilibria which can, under specific conditions, also represent more "natural" NE solutions. Section 2 of this paper summarizes the motivations behind this study in relation to the reviewed literature.

Ideally, any mathematical model of equilibrium in capacitated networks should be accompanied by clearly stated principles of equilibrium. Given that Wardrop's first principle, as presented in Definition 1, does not strictly define NE, it is required to redefine Wardrop's first principle for capacitated networks. This is one task in this study. This paper contributes to the literature in the following dimensions:

(a) It presents a complementarity traffic equilibrium model with capacity constraints. The original form of the model represents the entire set of $\mathrm{NE}$ and special cases of it represent sub-sets of equilibria;

(b) The complementarity model is then used to redefine Wardrop's first principle for capacitated networks. The extended Wardrop's principle determines the set of NE for capacitated networks.

The paper is organized into the following sections. A review of the relevant literature is presented in Section 2. Section 3 presents some preliminary discussion to set the stage for Section 4 which describes UE in capacitated networks. Section 5 provides the complementarity equilibrium model and the extended Wardrop's principle for capacitated networks. Numerical experiments are performed in Section 6. Conclusions are provided in Section 7.

\section{Literature review}

The importance of including link capacities in traffic equilibrium models lies in the unrealistic form of the volume-delay functions that are usually assumed to be polynomial with degrees that can be determined from real data [7]. It is argued that because volume-delay functions are finite, nonlinear, positive, and strictly increasing with flow, they can lead to arbitrarily large travel times [8]. In reality, however, links have limited capacity and cannot carry flows that are larger than their capacities. When link flows exceed certain limits, travel time becomes meaningless [9]. Larsson and Patriksson [10], Marcotte et al. [6] and Correa et al. [11] all advocated the necessity of including link capacities as a way to ensure that the resulting link travel times are consistent with real-life situation. The following discussion tries to shed light on different aspects of flow equilibria and link capacity considerations.

One aspect of the equilibrium flow pertains to the relationship between the flow and its day-to-day dynamics. Zhang et al. [12] investigated the equivalence of stationary day-to-day link flow pattern and traffic network equilibrium. They do this for both static and dynamic network problems, under three costresponsive mechanisms for route choice. Their analysis does not require complete information, homogeneous user behavior or monotonicity of the user travel cost function for links. They asserted that this result is not "obvious", because link flow stationarity does not necessarily imply path flow stationarity, and that multiple equilibria may exist. They emphasized that this result:

1. Approves that the link flow pattern monitoring to be more suitable compared to the more difficult path flow monitoring;

2. Provides a base for Intelligent Transportation Systems (ITS) directive.

Following Zhang et al. [12], Yang and Zhang [13] showed that five special categories of route adjustment processes in the literature lead to a rational behavior adjustment process. They, then, based on these results, concluded that one may now accept the equivalency of the stationary link flow pattern for the day-to-day traffic dynamics and UE flow as a "sound theory".

Using Lyapunov theorem, Smith [14] presented a dynamical model that converges to a set of Wardrop equilibria over time, regardless of the starting flow condition, assuming monotone and smooth travel cost function. The motivation behind his study includes the interests that the operators have, regarding the stability of flow over time, the possible multiplicity of the solutions to the equilibrium flow problems for the different starting points, and if there exist more than one equilibrium, which one is better, and how one may drive the system to the better equilibrium. His analysis is valid when there are no explicit capacity restrictions, and he exploited the concept of swapping flow from the more expensive route of an $\mathrm{O} / \mathrm{D}$ pair to a less costly alternative route of that $\mathrm{O} / \mathrm{D}$. Jin [15], in briefly reviewing the evolution of the traffic assignment problem from early 1950 's to 2005 , analyzed the asymptotic stability of the fixed demand UE in static transportation networks for two non-monotone traffic assignment models of Smith [14] and Jin [16] representing the user's day-to-day behavior, and showed that both models 
present consistent results, with the latter being simpler than the former, particularly when dealing with road network traffic dynamics and capacity constraints. (A discussion of the conventional Dynamic Traffic Assignment (DTA) models and the existence and uniqueness of their solutions, particularly, in regard to Wardropian solution, may be found in Szeto and Lo [17].)

Marcotte et al. [6] discussed the concept of strategy to model traffic assignment (rather than path selection in a network). They noted that finiteness of link capacities should not be ignored in traffic assignment. They defined a variational inequality relationship for strategy-based equilibria, and presented algorithms to turn strategies to link flows. The concept of strategy, previously defined for transit network assignment, is used for capacitated acyclic networks. However, their model does not guarantee a unique solution.

Nasri and Sosa [18] reformulated the generalized NE problems as an equilibrium problem, and showed its existence result without resort to the monotonicity of the objective function. Interpretation of UE flow in transportation networks, as non-cooperative games, has been introduced a long time ago (see $[19,20]$ ). Ever since, game theory has been applied to describe users' behaviors. A more recent investigation on the interaction between two (or more) vehicles, using game theory, has been performed by Levinson [21] who showed that, for a two-player game, congestion occurs based on the players' relative valuations of early/ late arrivals and journey delay. In this game, congestion pricing is viewed as a cooperation mechanism to minimize total costs if returned to the players. For a three-player game, congestion becomes a negative externality for the players. Zou and Levinson [22] further discussed a multi-agent model to show congestion and pricing effects on traveler behaviors and network equilibrium. They solved the $N$-player game (for $N \leq 7$ ) for Nash equilibria, when exist. Han et al. [23] presented a model which includes a term indicating the endogenous interaction among self-interest individuals making choices in a capacity-constrained urban system. The endogenous interaction term is imbedded within a discrete choice model representing the strategic behavior of the individuals with different degrees of rationality and levels of experience. These $N$-player $J$-option game models are also linked to the actions of a controlling agent providing information for various objectives' optimization, resulting in different equilibria.

Anshelevich et al. [24] investigated the effect of the strategic behavior on the network design problem. They found that the set of stable solutions (the Nash equilibria) differ from that of the central optimum. They also discussed the quality of the best NE, using the price of stability measure defined as the ratio of the cost of NE solution over that of the optimum network.

The existing literature contains several types of game theoretic concepts in connection with the network flows. Li et al. [25] have noted an interesting Stackelberg game in traffic flow, in which the operator of the network is the leader (providing information on travel costs) and the users (drivers) of the network are the followers who choose paths based on the available information. This game explores the competitive and cooperative relationship between User and System Equilibria (UE and SE) of Wardrop's first and second principles, respectively. It persuades the competitive users to act more cooperatively. They reported several other games concerning traffic flow, in which both competition and cooperation exist, which shows a wide range of interest in visualizing and formulating traffic flow problems as games.

Schulz and Stier Moses [26], noting that ITS aim to lead the actual traffic to the system optimum flow, intended to show that the cost of working with user equilibria, instead of system optima, is limited for a wide range of network classes. They emphasized, despite the fact that link capacities lead to multiple equilibria, the good news is that UE flow is close to the system optimum. They introduced user equilibria in capacitated network, and showed that the worst ratio of the total travel times of the best UE over SE does not change with the inclusion of capacities in the problem. Moreover, they presented bounds as the performance guarantee of UE to constrained SE. In the latter SE, users are assigned to routes that are not "too long".

There is a debate as to what equilibrium should be left prevailing in a network. As an operator of the system, a good flow pattern is one which is "fair" and "efficient". Fair in the sense of travel times experienced by different users, and efficient in the sense of the total travel times spent in the network (of interest to the operator of the system). However, these two objectives may prove to be conflicting in real networks: $\mathrm{UE}$ is in a sense fair, but not efficient (relative to $\mathrm{SE}$ ), because all users of the same O/D experience the same travel cost. Nevertheless, this fairness may not extend to the users of different O/D's. On the other hand, SE is efficient (with the minimum total travel cost), but may not be fair in the sense mentioned above. Moreover, in SE, some users may experience excessively long O/D travel times. Jahn et al. [27] aimed to establish a flow pattern, by taking advantage of "reactive guidance", which trades off these two objectives: being efficient without losing the fairness. They defined several unfairness measures, but used normal (length) unfairness, in relation to each O/D, as the maximum ratio of the distance traveled in different paths over the distance of the shortest travel time path for the same $\mathrm{O} / \mathrm{D}$ pair, $\alpha, \alpha \geq 1$. That is, an $\mathrm{O} / \mathrm{D}$ path is deemed "feasible" if its distance is less than or equal to $\alpha$ times, the shortest travel time path for that $\mathrm{O} / \mathrm{D}$ pair. They, then, minimized the total travel 
times of the network subject to the conservation of flow and the flow non-negativity constraints, within such feasible path sets for the O/D pairs, and called it the Constrained System Optimum (CSO) problem. Relaxation of the normal fairness constraint would turn CSO into SE problem.

Other authors exploiting some other notions, such as reliability, also introduced similar concepts. For example, Poorzahedy and Bushehri [28] presented a pessimistic equilibrium flow problem, which is a generalization of UE problem, in which link travel cost is the sum of the average travel time and a measure of its variability $\left(k_{\alpha} \times\right.$ standard deviation of average travel time), to make sure that the traveler reaches the destination within $100 \alpha$ percent of the times. They showed that this flow may generate less total travel time than the UE flow. Noting that route guidance may reduce congestion, Jahn et al. [27] tried to construct such efficient-fair flow patterns in networks by exploiting in-vehicle reactive route guidance devices which rely upon current traffic condition with no future pattern prediction. They also mentioned the limitations of their model, as dealing with static flow, in which all drivers follow the route guidance recommendations.

Among other works related to equilibrium flow, the one brought in by Tobin and Friesz [29] may be of value to this discussion. Some existing methods, because of lacking the necessary conditions for application, may not be applicable to the equilibrium traffic flow problems. Examples are the application of the existing Sensitivity Analysis (SA) methods which require uniqueness of the solution to the problem. Tobin and Friesz [29] presented an equivalent restricted problem to the Equilibrium Traffic Assignment Problem (ETAP), having the desired properties. They showed that the derivatives of the equilibrium link flow, with respect to the perturbations of the cost functions and trip table found by the application of the existing SA methods, are equivalent to those of the original unrestricted ETAP. The importance of such findings bears this sign that despite the apparent irregularities attached to the equilibrium flow problem, it is friendlier than it may seem.

There are two prominent strategies for incorporating link capacities in equilibrium models. The first strategy is to redefine the volume-delay function, so that travel time tends to infinity as link flow reaches link capacity [8,30,31]. Although this strategy reduces the capacity constrained problem to an unconstrained problem, it creates various other issues such as causing unrealistically high travel times, devious routing of trips [32] and numerical difficulties near capacity flow [33]. The second strategy, which is more widely used, incorporates capacity constraints in the model by setting the general travel time equal to the sum of the actual link travel time and the dual variable associated with the capacity constraint of the link [34]. The dual variables in this strategy are interpreted by Daganzo [35], as the point queue delays incurred at the entrance of each capacitated link. The use of dual variables has been the motivation behind many algorithms for finding the equilibrium solution of capacitated networks. These methods can be divided into exterior penalty function methods $[34,36]$, inner penalty function methods [33,37] and Lagrange multiplier methods $[9,38,39]$.

Marcotte et al. [6] argued that the use of the dual variable of the capacity constraint as queuing delay is controversial, as it leads to capturing only a subset of equilibria. Instead, they presented the extended Wardrop's first principle for capacitated networks (Definition 1), and used the concept of hyperpath assignment in which users are assigned to strategies that provide a set of sub-paths at every node. The model is then formulated as a variational inequality problem in which the finite capacity assumption is assumed to be proxy for travel delay. A partial linearization method and a projection method are then presented to solve the variational inequality model.

Correa et al. [11] defined Wardrop's first principle for capacitated networks such that no OriginDestination (OD) pair has an unsaturated path (path with excess capacity) with a strictly smaller cost than any used path for that pair. They emphasized that their definition of Wardrop's first principle is not strictly equivalent to NE in capacitated networks. They regarded the addition of the capacity constraints to the BMW optimization problem as the natural method of solving for UE. After defining the Beckmann et al. [2] problem with capacity constraints and formulating price-of-anarchy, Correa et al. [11] showed that the best obtained equilibrium in capacitated networks is as efficient as the equilibrium in uncapacitated networks.

The above review of the literature shows the wide range of interests in the study of equilibrium flow. They include the theoretical aspects of investigating relations between UE and other equilibrium concepts, such as $\mathrm{NE}$ or SE, or the relations between UE and games, or the relations between "fair" and "efficient" flow patterns; and the practical aspects of the development of strategy-based traffic assignment in a network, and the provision of a base for ITS directives.

It also shows that there are currently no available equilibrium models, for capacitated networks, that represent an NE [6,11]. Developing such a model requires redefining Wardrop's first principle. Moreover, exploring the relationship between NE and Wardrop equilibrium is essential for capacitated networks, as others have already investigated this relationship for uncapacitated networks [40]. In particular, it is re- 
quired to identify the conditions under which the two equilibria are equivalent in capacitated networks.

The motivations behind this study, therefore, are listed as follows:

1. Researchers noted that finiteness of link capacities should not be ignored in traffic assignment [40];

2. There is a need to explore the existence of multiple equilibria to identify the more efficient and fairer solution to strive for it;

3. It is important to explore the relations between UE and other equilibrium concepts, such as NE, for being used as selection criteria in the choice between different user equilibria, and take measures to guide flow patterns toward the preferred one;

4. It is fruitful to investigate the applicability of the existing analytical models, developed for the uncapacitated UE flow problem, to the capacitated UE flow problem.

The discussion presented in the next section sets the stage for that of the UE in capacitated networks.

\section{UE in uncapacitated networks}

Consider a directed graph $G(N, A)$ where $N$ is the set of nodes and $A$ is the set of links. Let $R \subseteq N$ denote the set of origin nodes, and $S \subseteq N$ denote the set of destination nodes. Each OD pair with origin node $r \in R$ and destination node $s \in S$ is hereafter denoted by $r s$. Let $q_{r s}>0$ denote the travel demand from origin $r$ to destination $s$, and $K_{r s}$ denote the set of paths from origin $r$ to destination $s$. Let $f_{k}$ denote the traffic flow on path $k$ and $f$ be the path flow vector. Let $x_{a}$ denote the traffic flow on link $a \in A, x$ be the link flow vector, and $c_{a}$ denote the traffic flow capacity of link $a$. Let $t_{a}\left(x_{a}\right)$ denote the volume-delay function for link $a$ which is continuous and non-decreasing, and $T_{k}(f)$ be the travel time of path $k$. Let $\delta_{a k}$ be the link-path incidence parameter which is equal to 1 , if link $a$ is in path $k$, and 0 otherwise, and let $A^{k}=\left\{a \in A \mid \delta_{a k}=1\right\}$.

Using the above notation, the link flows are calculated, using Eq. (1), and the path travel times are calculated, using Eq. (2).

$$
\begin{array}{ll}
x_{a}=\sum_{r s} \sum_{k \in K_{r s}} \delta_{a k} f_{k}, & \forall a \in A, \\
T_{k}(f)=\sum_{a \in A} \delta_{a k} t_{a}\left(x_{a}\right)=\sum_{a \in A^{k}} t_{a}\left(x_{a}\right) & \forall k \in K_{r s}, r s .
\end{array}
$$

The flow $f$ is a feasible flow vector if all path flows are non-negative and if the sum of path flows for each OD pair is equal to the demand of that OD pair. These two conditions are applied in Eq. (3):

$$
\sum_{k \in K_{r s}} f_{k}=q_{r s} \quad \forall r s
$$

$$
f_{k} \geq 0 \quad \forall k \in K_{r s}, r s
$$

A feasible flow that satisfies Eqs. (1), (2) and (3) is in NE, if no traveler can take another path with a lower travel time. $\mathrm{NE}$ is mathematically presented in Definition 2. (A similar definition is provided in page 73 of Patriksson [7], which used the term "user optimized" instead of NE. A version of Definition 2 is also presented in Roughgarden and Tardos [41], without imposition of condition $f_{k}>0$, which might have been overlooked. Note that when $f_{k}=0$, for $\theta=0$ in Eq. (4) below, we have $T_{k}(f) \leq T_{l}(f)$, which is not necessarily true for each $l$.)

Definition 2. $\quad N E$ : A feasible network flow $f$ is an $\mathrm{NE}$, if for each pair $r s$ and $k, l \in K_{r s}$ with $f_{k}>0$, and each $\theta \in\left[0, f_{k}\right]$ we have:

$$
T_{k}(f) \leq T_{l}\left(f-\theta u^{k}+\theta u^{l}\right)
$$

where $u^{k}$ is a unit vector in the path space with element $k$ equal to 1 , and all other elements equal to 0 .

Definition 2 indicates that no user can benefit from changing paths from $k$ to $l$ under NE. Lemma 1 shows that Wardrop equilibrium is equivalent to $\mathrm{NE}$ for uncapacitated networks.

Lemma 1. Wardrop's equilibrium: A feasible flow $f$ is in equilibrium, if and only if for each pair $r s$ and each $k, l \in K_{r s}$ with $f_{k}>0$ we have $T_{k}(f) \leq T_{l}(f)$.

Proof. See Roughgarden and Tardos's work [41] and the references cited therein for an equivalent version of this lemma.

Whereas Lemma 1 presents a clear proof of equilibrium for uncapacitated networks, the process of defining Wardrop's first principle for capacitated networks is not clear. Hence, we define here the concept of attraction potential, which is the excess capacity of some path $k \in K_{r s}$ that can be filled up with extra flow from some path $l \in K_{r s}$ such that the transferred flow experiences a lower travel time. More formally, let $\Delta_{k}(f)>0$, if there exists a path $l \in K_{r s}$ with $f_{l}>0$ such that $T_{l}(f)>T_{k}(f)$. Otherwise, $\Delta_{k}(f)=0$. In other words, we have:

$$
\Delta_{k}(f)= \begin{cases}+, & \text { if } \exists l \in K_{r s} \text { with } f_{l}>0, T_{l}(f)>T_{k}(f) \\ 0, & \text { otherwise }\end{cases}
$$

(The authors track the concept of "swapping flow from the more expensive route of an $\mathrm{O} / \mathrm{D}$ pair to a less costly alternative route of that $\mathrm{O} / \mathrm{D}$ " back to as early as the work of Smith [14]. See, also [12,13] in this regard.) We now express the equilibrium in Lemma 2, using the concept of attraction potential. 
Lemma 2. The feasible flow $f$ is in equilibrium, if and only if for each pair $r s$ and $k \in K_{r s}$, we have $\Delta_{k}(f)=0$.

\section{Proof}

$\Rightarrow$ If $\Delta_{k}(f)=0$ for at least one pair $r s$ and one $k \in K_{r s}$, then there exists a path $l$ with $f_{l}>0$ and $T_{l}(f)>T_{k}(f)$ which, according to Lemma 1 , contradicts $f$ to be in equilibrium.

$\Leftarrow$ If $\Delta_{k}(f)=0$ for each pair $r s$ and each $k \in K_{r s}$, then for each $l \in K_{r s}$ with $f_{l}>0$, we have $T_{l}(f) \leq$ $T_{k}(f)$ which, according to Lemma 1 , indicates that $f$ is in equilibrium.

We use Lemmas 1 and 2 to show, in Proposition 1 , that the travel time of all used paths of each OD pair $r s$ is at most equal to $u_{r s}$.

Proposition 1. The feasible flow $f$ is in equilibrium, if and only if for each pair $r s$ there exists a $u_{r s}$ such that for each $k \in K_{r s}$ we have:

$$
\begin{array}{ll}
T_{k}(f) \leq u_{r s} & \text { if } f_{k}>0, \\
T_{k}(f) \geq u_{r s} & \text { if } f_{k}=0 .
\end{array}
$$

Proof. Assume that $f$ is in equilibrium. For each pair $r s$, given that $q_{r s}>0$, there exists at least one path with a positive flow. By setting $u_{r s}=$ $\max _{k \in K_{r s}: f_{k}>0} T_{k}(f)$, or for short $T_{k^{*}}(f)$, Eq. (5-1) becomes obvious. Moreover, based on Lemma 1, Eq. (5-2) is satisfied since for each $k \in K_{r s}$, given that $f_{k^{*}}>0$, we have:

$$
T_{k}(f) \geq T_{k^{*}}(f)=u_{r s} .
$$

Assume that $f$ is not in equilibrium. Based on Lemma 2, there exists a $k \in K_{r s}$ such that $\Delta_{k}(f)>0$. Hence, there exists a path $l \neq k$ in $K_{r s}$ for which $f_{l}>0$ and $T_{l}(f)>T_{k}(f)$. On the other hand, given Eq. (5-1), we have $T_{l}(f) \leq u_{r s}$, and given Eq. (5-2), we have $T_{k}(f) \geq u_{r s}$ and $T_{l}(f) \leq T_{k}(f)$, which implies a contradiction.

Proposition 2 is readily derived from Proposition 1 .

Proposition 2. The feasible flow $f$ is in equilibrium, if and only if $f$ is the solution of the following non-linear system:

$$
\begin{array}{ll}
\left(T_{k}(f)-u_{r s}\right) f_{k}=0 & \forall k \in K_{r s}, r s, \\
\left(T_{k}(f)-u_{r s}\right) \geq 0 & \forall k \in K_{r s}, r s .
\end{array}
$$

Proof. Given Proposition 1, it is sufficient to show that Eq. (5) is equivalent to Eq. (6), which is obvious.
Proposition 2 describes Wardrop's first principle or the following traffic equilibrium law: At equilibrium in an uncapacitated network, the travel times of all used paths are equal to each other, and smaller than or equal to the travel time of all unused paths [42]. The next section proceeds by extending the proofs of Section 3 to define the equilibrium conditions in capacitated networks.

\section{Equilibrium flow in capacitated networks}

In capacitated networks, the feasible flow is defined such that Eqs. (1), (2) and (3) are all satisfied in addition to the condition that the flow of each link must not be larger than the capacity of that link. Hence, the set of feasible flows is defined as:

$$
\begin{array}{ll}
\sum_{k \in K_{r s}} f_{k}=q_{r s} & \forall r s, \\
f_{k} \geq 0 & \forall k \in K_{r s}, r s, \\
x_{a} \leq c_{a} & \forall a \in A, \\
x_{a}=\sum_{r s} \sum_{k \in K_{r s}} \delta_{a k} f_{k} & \forall a \in A .
\end{array}
$$

For a feasible flow $f$, OD pair $r s$, and $k, l \in K_{r s}$, we can transfer a part of flow on path $k$ to path $l$, if the following two conditions are satisfied:

Condition 1: $f_{k}>0$,

Condition 2: $x_{a}<c_{a} \quad \forall a \in A^{l} \backslash A^{k}$.

Condition 1 ensures that path $k$ has a non-negative flow to be transferred, and Condition 2 ensures that path $l$ has some non-zero excess capacity that could be filled with the transferred flow from path $k$. To represent mathematically the concept of flow transferability, we define $\varphi_{k l}(f)$ as the maximum flow that can be transferred from path $k$ to path $l$, which is calculated as follows:

$$
\begin{aligned}
& \varphi_{k l}(f)=\min \left\{f_{k}, \min _{a \in A^{l} \backslash A^{k}} c_{a}-x_{a}\right\} \\
& \forall k, l \in K_{\text {rs }}, r s .
\end{aligned}
$$

Definition of $\varphi_{k l}(f)$, which is an extension of the concept of attraction potential in Section 3, allows us to extend Definition 2 of NE to capacitated networks as follows. A feasible flow is in equilibrium, if no user (or group of users) can reduce his/her (their) travel time by changing to another path which is not already at capacity. This definition is presented mathematically as follows.

Definition 3. The feasible flow $f$ is in equilibrium, if for each pair $r s$ and $k, l \in K_{r s}$ with $\varphi_{k l}(f)>0$, and any $\theta \in\left[0, \varphi_{k l}(f)\right]$, we have: 


$$
T_{k}(f) \leq T_{l}\left(f-\theta u^{k}+\theta u^{l}\right)
$$

Definition 3 indicates that no user group can benefit from changing paths from $k$ to $l$. Lemma 3 shows the equilibrium conditions in capacitated networks, using the concept of flow transferability.

Lemma 3. The feasible flow $f$ is in equilibrium, if and only if for each pair $r s$ and $k, l \in K_{r s}$ with $\varphi_{k l}(f)>$ 0 , we have $T_{k}(f) \leq T_{l}(f)$.

\section{Proof}

$\Rightarrow$ For each $k, l \in K_{r s}$, if $\varphi_{k l}(f)>0$, then for $\theta=0$, and according to Definition 3 , we have $T_{k}(f) \leq$ $T_{l}(f)$.

$\Leftarrow$ Assume that $T_{k}(f) \leq T_{l}(f)$ for each $k, l \in K_{r s}$ with $\varphi_{k l}(f)>0$. Hence, for any $\theta \in\left[0, \varphi_{k l}(f)\right]$, we have:

$$
\begin{aligned}
& T_{l}\left(f-\theta u^{k}+\theta u^{l}\right)=\sum_{a \in A^{l} \backslash A^{k}} t_{a}\left(x_{a}+\theta\right) \\
& +\sum_{a \in A^{l} \cap A^{k}} t_{a}\left(x_{a}\right) .
\end{aligned}
$$

Given that $t_{a}$ is non-decreasing and $\theta \geq 0$, we have:

$$
\begin{gathered}
T_{l}\left(f-\theta u^{k}+\theta u^{l}\right) \geq \sum_{a \in A^{l} \backslash A^{k}} t_{a}\left(x_{a}\right) \\
+\sum_{a \in A^{l} \cap A^{k}} t_{a}\left(x_{a}\right)=T_{l}(f) \geq T_{k}(f) .
\end{gathered}
$$

Hence, flow $f$ is in equilibrium.

Lemma 3 can also be presented as follows. Flow $f$ is in equilibrium, if and only if:

$$
T_{k}(f) \leq \min _{l \in K_{r s} \mid \varphi_{k l}(f)>0} T_{l}(f) \quad \forall k \in K_{r s}, r s .
$$

For a feasible flow $f$, path $k \in K_{\text {rs }}$ has some attraction potential to improve travel time, or $\Delta_{k}(f)>0$, if there exists a path $l \in K_{r s}$ such that $\varphi_{l k}(f)>0$ and $T_{l}(f)>$ $T_{k}(f)$. Otherwise, $\Delta_{k}(f)=0$. In other words:

$$
\Delta_{k}(f)= \begin{cases}+, \quad \text { if } \exists l \in K_{r s} \text { with } \varphi_{l k}(f)>0, \\ & T_{l}(f)>T_{k}(f) \\ 0, & \text { otherwise }\end{cases}
$$

Using the definition of attraction potential above, we can rewrite Lemma 3 as Lemma 4 below:

Lemma 4. The feasible flow $f$ is in equilibrium, if and only if for each pair $r s$ and $k \in K_{r s}$ we have $\Delta_{k}(f)=0$.

\section{Proof}

$\Rightarrow$ Given a pair $r s$ and some $k \in K_{r s}$, if we have $\Delta_{k}(f)>0$, then there exists a path $l \in K_{r s}$ with $\varphi_{l k}(f)>0$ and $T_{l}(f)>T_{k}(f)$, which contradicts $f$ to be in equilibrium according to Lemma 3 .

$\Leftarrow$ If for each pair $r s$ and $k \in K_{r s}$ we have $\Delta_{k}(f)=0$, then for each $l \in K_{r s}$ with $\varphi_{l k}(f)>0$, we have $T_{l}(f) \leq T_{k}(f)$ which, according to Lemma 3 , indicates that $f$ is in equilibrium.

Using Lemma 2, we show, in Proposition 3, that the cost of any used path for each OD pair $r s$ is at most equal to $u_{r s}$. For ease of presentation, in what follows, we omit the argument $(f)$ from $T_{k}(f), \Delta_{k}(f)$, and $\varphi_{l k}(f)$.

Proposition 3. The feasible flow $f$ is in equilibrium, if and only if for each pair $r s$ there exists some $u_{r s}$ for each $k \in K_{r s}$, which satisfies the following:

$$
\begin{aligned}
& T_{k} \leq u_{r s} \quad \text { if } f_{k}>0, \\
& T_{k} \geq u_{r s} \quad \text { if } \Delta_{k}>0 .
\end{aligned}
$$

\section{Proof}

$\Rightarrow$ Assume that $f$ is the equilibrium flow. For each pair $r s$, given that $q_{r s}>0$, there exists at least one path with a positive flow. By letting $u_{r s}=\max _{k \in K_{r s}: f_{k}>0} T_{k}$, Eq. (9-1) becomes obvious. Moreover, given that $f$ is the equilibrium flow and given Lemma 4 , there exists no path $k$ with $\Delta_{k}>0$. Hence, Eq. (9-2) has no merit.

$\Leftarrow$ Assume $f$ is not in equilibrium, which means there exists a path $k \in K_{r s}$ with $\Delta_{k}>0$. Hence, there also exists a path $l \neq k$ in $K_{r s}$ with $\varphi_{l k}>0$ and $T_{l}>T_{k}$. Given $\varphi_{l k}>0$, we know that $f_{l}>0$, which according to Eq. (9-1) gives $T_{l} \leq u_{r s}$. On the other hand, $\Delta_{k}>0$ and Eq. (9-2) indicate that $T_{k} \geq u_{r s}$. Hence, we have $T_{l} \leq T_{k}$, which contradicts the initial assumption.

Proposition 3 can be presented in mathematical form as a non-linear system, as shown in Proposition 4.

Proposition 4. The feasible flow $f$ is in equilibrium, if and only if $f$ is the solution of the following system:

$$
\begin{array}{ll}
\left(T_{k}+\beta_{k}-u_{r s}\right) f_{k}=0 & \forall k \in K_{r s}, r s, \\
T_{k}+\beta_{k}-u_{r s} \geq 0 & \forall k \in K_{r s}, r s, \\
\Delta_{k} \beta_{k}=0 & \forall k \in K_{r s}, r s, \\
\beta_{k} \geq 0 & \forall k \in K_{r s}, r s .
\end{array}
$$

Proof. If $f$ is a feasible flow, given Proposition 3 , it is sufficient to show that Eq. (9) is equivalent to Eq. (10). 
$\Rightarrow$ For each pair $r s$ and $k \in K_{r s}$ :

If $f_{k}>0$ and $\Delta_{k}>0$, Eq. (9) indicates that $T_{k}=u_{r s}$. Hence, Eq. (10) holds for $\beta_{k}=0$;

If $f_{k}=0$ and $\Delta_{k}>0$, then Eq. (9-2) indicates that $T_{k} \geq u_{r s}$. Hence, Eq. (10) holds for $\beta_{k}=0$; If $f_{k}>0$ and $\Delta_{k}=0$, then Eq. (9-1) indicates that $T_{k} \leq u_{r s}$. Hence, Eq. (10) holds for $\beta_{k}=$ $u_{r s}-T_{k} \geq 0$;

If $f_{k}=0$ and $\Delta_{k}=0$, then Eq. (10) holds for $\beta_{k}=\max \left\{u_{r s}-T_{k}, 0\right\}$.

$\Leftarrow$ For each $r s$ and $k \in K_{r s}$ :

If $f_{k}>0$, then Eq. (10-1) indicates $T_{k}+\beta_{k}=u_{r s}$, which, according to Eq. (10-4), proves Eq. (9-1); If $\Delta_{k}>0$, then Eq. (10-3) indicates $\beta_{k}=0$, which, according to Eq. (10-2), proves Eq. (9-2).

The non-linear system in Proposition 4 requires a proper definition of the values of $\beta_{k}$ and $\Delta_{k}$, which can be cumbersome. Instead, we present a complementarity equilibrium model [43] that is equivalent to the non-linear system in Proposition 4, without the complications of defining the variables.

\section{A complementarity model for traffic equilibrium}

\subsection{Mathematical model}

A complementarity model is presented in this section to describe the equilibrium flow in capacitated networks. Let $\lambda_{a}^{k}$ denote the penalty of using a saturated link $a$ (for which $x_{a}=c_{a}$ ) in path $k$. Clearly, $\lambda_{a}^{k}$ is 0 for unsaturated links (for which $x_{a}<c_{a}$ ). Let $t_{a}(x)+\lambda_{a}^{k}$ be the generalized travel time of link $a$ in path $k$, which is the sum of the actual link travel time and penalty of using a capacitated link.

Definition 4. (Extended Wardrop's first principle for capacitated networks). At equilibrium, for each OD pair, the generalized travel time on all used paths are equal, and are less than or equal to the generalized travel time on all unused paths. Moreover, for any node $i$ and $j$, the travel time on any sub-path from $i$ to $j$ of any path with positive flow is less than or equal to the travel time of any unsaturated sub-path from $i$ to $j$ of any other path. We call this equilibrium path-level link penalty equilibrium.

The reason for imposing the above condition on any pair of nodes $i$ and $j$ is later explained in this section and visually depicted in the numerical experimentation section.

The feasible flow $f$ satisfies the extended complementarity model of traffic equilibrium, if and only if the following non-linear conditions are satisfied:

$$
\begin{aligned}
& \left(\sum_{a \in A} \delta_{a k}\left(t_{a}(x)+\lambda_{a}^{k}\right)-u_{r s}\right) f_{k}=0 \quad \forall k \in K_{r s}, r s, \\
& \sum_{a \in A} \delta_{a k}\left(t_{a}(x)+\lambda_{a}^{k}\right)-u_{r s} \geq 0 \quad \forall k \in K_{r s}, r s, \\
& \left(\sum_{a \in A} \delta_{a k}^{i j} t_{a}(x)-\sum_{a \in A} \delta_{a l}^{i j} t_{a}(x)\right) \\
& \times\left(\prod_{a \in A: \delta_{a l}^{i j}=1}\left(c_{a}-x_{a}\right)\right) f_{k} \leq 0 \quad \forall k, l \in K_{r s}^{i j}, i j, r s \\
& \left(c_{a}-x_{a}\right) \lambda_{a}^{k}=0 \quad \forall a \in A, \quad k \in K_{r s}, r s, \\
& \lambda_{a}^{k} \geq 0 \quad \forall a \in A, \quad k \in K_{r s}, r s,
\end{aligned}
$$

where:

$\lambda_{a}^{k}=$ The penalty of using saturated link $a$ (for which $\left.x_{a}=c_{a}\right)$ in path $k$,

$K_{r s}^{i j}=\left\{k \in K_{r s} \mid \exists\right.$ a sub-path in $k$ that connects node $i$ to node $j\}$,

$\delta_{a k}^{i j}= \begin{cases}1, & \text { if } a \text { is in a sub-path of } k \text { that connects } \\ & \text { node } i \text { to node } j \\ 0, & \text { otherwise. }\end{cases}$

We proceed by proving that the feasible flow is the equilibrium flow, if and only if it satisfies the complementarity Model (11). Proposition 5 and Proposition 6 present the necessity and sufficiency proofs, respectively. For simplicity of presenting the proofs, let:

$\bar{c}_{a}=$ The remaining capacity of link $a=c_{a}-x_{a}$,

$\bar{c}_{k}=$ The remaining capacity of path $k=\min _{a \in A^{k}} \bar{c}_{a}$,

$\bar{c}_{k}^{i j}=$ The remaining capacity of part of path $k$ that connects $i$ to $j=\min _{a \in A: \delta_{a k}^{i j}=1}\left\{c_{a}-x_{a}\right\}$,

$T_{k}^{i j}=$ The travel time of path $k$ from node $i$ to node $j=\sum_{a \in A} \delta_{a k}^{i j} t_{a}(x)$,

$\beta_{k}=\sum_{a \in A} \delta_{a k} \lambda_{a}^{k}$,

$\beta_{k}^{i j}=\sum_{a \in A} \delta_{a k}^{i j} \lambda_{a}^{k}$,

$k^{i j}=$ Part of path $k$ that extends from node $i$ to node $j$.

Proposition 5. The feasible flow $f$ is in equilibrium, if it satisfies the complementary Model (11).

Proof. Given Proposition 4, it is sufficient to show that Model (11) implies Model (10). 
Let $f, \lambda$, and $u$ be the solution of Model (11). Then, $f, u$, and $\beta_{k}=\sum_{a \in A} \delta_{a k} \lambda_{a}^{k}$ comprise the solution of Model (10), such that Eqs. (11-1) and (112) imply Eqs. (10-1) and (10-2), and Eq. (11-5) implies $\beta_{k} \geq 0$. Hence, it is sufficient to show that Eq. (10-3) is satisfied, as well.

Assume the opposite is true and there exists $a \in$ $K_{r s}$, such that $\beta_{k}>0$ and $\Delta_{k}>0$. The latter $\left(\Delta_{k}>0\right)$ implies that there exists a path $l \in K_{r s}, l \neq k$, for which the following two conditions hold:

$$
\varphi_{l k}>0 \text { and } T_{l}>T_{k} .
$$

Condition $\varphi_{l k}>0$ implies that $f_{l}>0$ and $x_{a}<c_{a}$ for any $a \in A^{k} \backslash A^{l}$.

Condition $T_{l}>T_{k}$ implies that there exist two nodes $i$ and $j$ with $k, l \in K_{r s}^{i j}$, such that $T_{l}^{i j}>T_{k}^{i j}$ and $\bar{c}_{k}^{i j}>0$. The latter condition leads to $\prod_{a \in A: \delta_{a k}^{i j}=1}\left(c_{a}-\right.$ $\left.x_{a}\right)>0$, which according to the result of the former condition (i.e. $f_{l}>0$ ), contradicts Eq. (11-3).

Proposition 6. If the feasible flow $\mathrm{f}$ is in equilibrium, then it should satisfy the complementarity Model (11).

Proof. Given Proposition 4, it is sufficient to show that Model (10) implies Model (11). Assume $f$ to be the equilibrium flow of Model (10). We first show that Eq. (11-3) is satisfied. Then, we show that all other conditions are satisfied, as well.

Condition (11-3) is satisfied:

Consider $f, \beta$, and $u$ to be the solution of Model (10) but Eq. (11-3) is not satisfied, which means that there exist nodes $i$ and $j$ and paths $k$ and $l$ in $K_{r s}^{i j}$, such that $\bar{c}_{l}^{i j}>0$ and $f_{k}>0$ and $T_{k}^{i j}>T_{l}^{i j}$.

Now consider path $p$ composed of the sub-paths $k^{r i}-l^{i j}-k^{j s}$. Given that $\varphi_{k p}>0$ (since we have $x_{a}<c_{a}$ in the unshared parts of the paths $k$ and $p$ ) and $T_{k}>T_{p}$, we have $\Delta_{p}>0$. Hence, we conclude from Eq. (10-2), for path $p$, that:

$$
\beta_{p} \geq u_{r s}-T_{p} \text {. }
$$

Given that $T_{k}>T_{p}$, we have:

$$
\beta_{p}>u_{r s}-T_{k}
$$

and given $f_{k}>0$ by Eq. (10-1), the following result could be obtained:

$$
\beta_{p}>\beta_{k} \text {. }
$$

Since we have $\beta_{k} \geq 0$, the above condition implies that $\beta_{p}>0$ and $\Delta_{p} \beta_{p}>0$, which contradicts Eq. (103). Hence, Condition Eq. (11-3) is satisfied. All other conditions of Model (11) are satisfied:

We now show that if $f$ is the equilibrium flow of Model (10), then the values $u_{r s}$ and $\lambda_{a}^{k}$ can be defined such that they satisfy all other conditions of Model (11) as well.
For each $r s$, we partition the set $K_{r s}$ into the following four sets:

$$
\begin{aligned}
& K_{r s}^{1}=\left\{k \in K_{r s} \mid f_{k}>0, \bar{c}_{k}=0\right\}, \\
& K_{r s}^{2}=\left\{k \in K_{r s} \mid f_{k}>0, \bar{c}_{k}>0\right\}, \\
& K_{r s}^{3}=\left\{k \in K_{r s} \mid f_{k}=0, \bar{c}_{k}=0\right\}, \\
& K_{r s}^{4}=\left\{k \in K_{r s} \mid f_{k}=0, \bar{c}_{k}>0\right\} .
\end{aligned}
$$

It is simple to show that:

$$
\begin{array}{ll}
T_{k} \leq T_{l} \quad \forall k \in K_{r s}^{1}, \quad l \in K_{r s}^{2}, \\
T_{k}=T_{l} \quad \forall k, l \in K_{r s}^{2}, \\
T_{k} \leq T_{l} \quad \forall k \in K_{r s}^{1} \cup K_{r s}^{2}, \quad l \in K_{r s}^{4},
\end{array}
$$

since there would, otherwise, exist some path $k$ with $\Delta_{k}>0$, violating equilibrium. For each $r s$, with $q_{r s}>$ 0 , we choose the value of $\hat{u}_{r s}$, as follows:

$$
\hat{u}_{r s} \begin{cases}=\min _{k \in K_{r s}^{2}} T_{k}, & \text { if } K_{r s}^{2} \neq \emptyset \\ \geq \max _{k \in K_{r s}^{1}} T_{k}, & \text { if } K_{r s}^{2}=\emptyset\end{cases}
$$

Note that $K_{r s}^{1} \cup K_{r s}^{2} \neq \emptyset$ since $q_{r s}>0$.

For each $a \in A$ and $k \in K_{r s}$, the values $\lambda_{a}^{k}$ are chosen to satisfy the following conditions:

$$
\begin{aligned}
& \lambda_{a}^{k}=0 \quad \text { if } \delta_{a k}=0 \text { or } \bar{c}_{a}>0, \\
& \lambda_{a}^{k}=M \quad \text { if } \delta_{a k}=1 \text { and } \bar{c}_{a}=0 \text { and } f_{k}=0, \\
& \sum_{a \in A} \delta_{a k} \lambda_{a}^{k}=\hat{u}_{r s}-T_{k} \quad \text { if } f_{k}>0, \\
& \lambda_{a}^{k} \geq 0,
\end{aligned}
$$

where $M$ is a big number.

We, now, first show that if $\hat{u}_{r s}$ and $\lambda_{a}^{k}$ are chosen according to Conditions (16)-(19), then all conditions of Model (11) would be satisfied as well. Then, we show that $\lambda_{a}^{k}$ can always be chosen such that Eqs. (16)-(19) are satisfied. Given Eqs. (16) and (19), it is clear that Eqs. (11-4) and (11-5) are satisfied. Condition (11-3) is also satisfied, as shown before. Hence, it is sufficient to show that Eqs. (11-1) and (11-2) are satisfied. To show this, we consider the two following scenarios with $K_{r s}^{2} \neq \emptyset$ and $K_{r s}^{2}=\emptyset$.

Scenario 1: $K_{r s}^{2} \neq \emptyset$. According to Eq. (15), we have $\hat{u}_{r s}=\min _{k \in K_{r s}^{2}} T_{k}$.

- For each $k \in K_{r s}^{1}$, Eqs. (12) and (13) imply that $\hat{u}_{r s} \geq T_{k}$; given that $\bar{c}_{k}=0$, there exists at least one saturated link in path $k$; and since $f_{k}>0$ and according to Eqs. (18), (11-1) and (11-2) are satisfied; 
- For each $k \in K_{r s}^{2}$, Eq. (13) implies $\hat{u}_{r s}=T_{k}$, and since $f_{k}>0$, Eq. (18) implies $\lambda_{a}^{k}=0$. Hence, Eqs. (11-1) and (11-2) are satisfied;

- For each $k \in K_{r s}^{3}$, since $f_{k}=0$ and $\bar{c}_{k}=0$, there exists at least one saturated link in path $k$. Hence, setting $\lambda_{a}^{k}=M$, Eqs. (11-1) and (11-2) would be satisfied;

- For each $k \in K_{r s}^{4}$, Eq. (14) implies that $\hat{u}_{r s} \leq T_{k}$. This condition, along with $f_{k}=0$ and $\lambda_{a}^{k} \geq 0$, satisfies Eqs. (11-1) and (11-2).

Scenario 2: $K_{r s}^{2}=\emptyset$. According to Eq. (15), we have $\hat{u}_{r s} \geq \max _{k \in K_{r s}^{1}} T_{k}$.

- For each $k \in K_{r s}^{1}$, it is clear that $\hat{u}_{r s} \geq T_{k}$. The rest of the proof is similar to Scenario 1 ;

- For each $k \in K_{r s}^{3}$, the proof is similar to Scenario 1;

- For each $k \in K_{r s}^{4}$, Eq. (14) implies $\hat{u}_{r s} \leq T_{k}$. The rest of the proof is similar to Scenario 1.

To complete the proof of the proposition, it is sufficient to show that $\lambda_{a}^{k}$ can be chosen so that (16)-(19) are satisfied.

We only need to find $\lambda_{a}^{k}$ for each $k \in K_{r s}^{1}$, because $\lambda_{a}^{k}=0$ for each $k \in K_{r s}^{2} \cup K_{r s}^{4}$ and $\lambda_{a}^{k}=M$ for each $k \in K_{r s}^{3}$ where $\delta_{a k}=1$, and $\bar{c}_{a}=0$. That is, we only need to find $\lambda_{a}^{k}$ for the paths with $f_{k}>0$, which have at least one saturated link $\left(x_{a}=c_{a}\right)$. According to the previous discussion, under both defined scenarios, for each $k \in K_{r s}^{1}$ we have $\hat{u}_{r s} \geq T_{k}$ or $\hat{u}_{r s}-T_{k} \geq 0$. It is now sufficient to choose $\lambda_{a}^{k} \geq 0$ for the saturated links that belong to path $k$ (i.e. $\bar{c}_{a}=0$ ) so that:

$$
\sum_{a \in A^{k}: \bar{c}_{a}=0} \delta_{a k} \lambda_{a}^{k}=\hat{u}_{r s}-T_{k} .
$$

Since path $k$ has at least one saturated link, the above equation has always a solution. This completes the proof.

\subsection{Properties of the complementarity equilibrium model}

Condition (11-3) in Model (11) is particularity important as it leads to the following three remarks.

Remark 1. Condition (11-3) is equivalent to the following condition:

$$
\begin{aligned}
& \sum_{a \in A} \delta_{a k}^{i j} t_{a}(x) \leq \sum_{a \in A} \delta_{a l}^{i j} t_{a}(x), \\
& \quad \forall k, l \in K_{r s}^{i j}, i j, r s,: f_{k}>0, \bar{c}_{l}^{i j}>0 .
\end{aligned}
$$

Proof. We first show that Eq. (11-3) implies Eq. (20). If $f_{k}>0$ and $\bar{c}_{l}^{i j}>0$, then $\prod_{a \in A: \delta_{a l}^{i j}=1}\left(c_{a}-x_{a}\right)>0$, which shows that Eq. (20) can be derived from Eq. (11-3).

We now show that Eq. (20) implies Eq. (11-3):
If $f_{k}=0$, then Eq. (11-3) is always satisfied; If $\bar{c}_{l}^{i j}=0$, then for one link $a \in A$ with $\delta_{a l}^{i j}=1$ we should have $x_{a}=c_{a}$, and hence:

$$
\prod_{a \in A: \delta_{a l}^{i j}=1}\left(c_{a}-x_{a}\right)=0 .
$$

That is, Eq. (11-3) is satisfied;

If $f_{k}>0$ and $\bar{c}_{l}^{i j}>0$, then it is clear that $\prod_{a \in \delta^{i j}=1}\left(c_{a}-x_{a}\right)>0$, and therefore Eq. (11-3) is $a \in A: \delta_{a l}^{i j}=1$

derived from Eq. (20).

Condition Eq. (20) represents the second part of Definition 4, which can also be defined as:

$$
T_{k}^{i j} \leq T_{l}^{i j} \quad \forall k, l \in K_{r s}^{i j}, i j, r s: f_{k}>0, \bar{c}_{l}^{i j}>0 .
$$

Remark 2. Condition (11-3) is not necessary for $i=$ $r$ and $j=s$, because it would be implied by Conditions (11-1), (11-2), (11-4) and (11-5). This is so because for $f_{k}=0$, Condition (11-3) is obvious. For $f_{k}>0$, Conditions (11-1) and (11-2) yield:

$$
\sum_{a \in A} \delta_{a k}^{r s}\left(t_{a}\left(x_{a}\right)+\lambda_{a}^{k}\right)=u_{r s} \leq \sum_{a \in A} \delta_{a l}^{r s}\left(t_{a}\left(x_{a}\right)+\lambda_{a}^{l}\right) .
$$

Since $\lambda_{a}^{k} \geq 0$, we obtain:

$$
\sum_{a \in A} \delta_{a k}^{r s} t_{a}\left(x_{a}\right) \leq \sum_{a \in A} \delta_{a l}^{r s}\left(t_{a}\left(x_{a}\right)+\lambda_{a}^{l}\right) .
$$

On the other hand, according to the feasibility condition of (7-3), we may write:

$$
\prod_{a \in A: \delta_{a l}^{r s}=1}\left(c_{a}-x_{a}\right) \geq 0 .
$$

Condition (3-11) holds if the left-hand-side of the latter relation becomes zero. For the non-zero value of this quantity, Conditions (11-4) yields $\lambda_{a}^{l}=0, \forall a \in A$ : $\delta_{a l}^{r s}=1$. As a result, we may write:

$$
\sum_{a \in A} \delta_{a l}^{r s}\left(t_{a}\left(x_{a}\right)+\lambda_{a}^{l}\right)=\sum_{a \in A} \delta_{a l}^{r s} t_{a}\left(x_{a}\right) .
$$

Then, according to Eq. (21), we may write:

$$
\sum_{a \in A} \delta_{a k}^{r s} t_{a}\left(x_{a}\right) \leq \sum_{a \in A} \delta_{a l}^{r s} t_{a}\left(x_{a}\right) .
$$

That is, Conditions (11-3) holds for $i=r$ and $j=s$.

Remark 3. Condition (11-3) is redundant when $\lambda_{a}^{k}=$ $\lambda_{a}$.

Proof. Consider the two paths $k$ and $l$ and the two nodes $i$ and $j$ that lie on those two paths. Assume that $f_{k}>0$ and $\bar{c}_{l}^{i j}>0$, from which it is clear that $\beta_{l}^{i j}=0$. Also consider a path $p$ which includes the sub-paths $k^{r i}-l^{i j}-k^{j s}$. We can conclude from Conditions (11$1)$ and (11-2) for paths $p$ and $k$ that: 


$$
\begin{aligned}
& T_{k}^{r i}+T_{k}^{i j}+T_{k}^{j s}+\beta_{k}^{r i}+\beta_{k}^{i j}+\beta_{k}^{j s}=u_{r s} \\
& T_{p}^{r i}+T_{p}^{i j}+T_{p}^{j s}+\beta_{p}^{r i}+\beta_{p}^{i j}+\beta_{p}^{j s} \geq u_{r s} .
\end{aligned}
$$

According to the assumption $\lambda_{a}^{k}=\lambda_{a}$ and the definition of path $p$, we have:

$$
\begin{array}{lll}
T_{p}^{r i}=T_{k}^{r i}, & T_{p}^{i j}=T_{l}^{i j}, & T_{p}^{j s}=T_{k}^{j s}, \\
\beta_{p}^{r i}=\beta_{k}^{r i}, & \beta_{p}^{i j}=\beta_{l}^{i j}=0, & \beta_{p}^{j s}=\beta_{k}^{j s} .
\end{array}
$$

Hence, we have $T_{k}^{i j}+\beta_{k}^{i j} \leq T_{l}^{i j} \Rightarrow T_{k}^{i j} \leq T_{l}^{i j}$ which implies that Conditions (11-3) is already satisfied and redundant.

We present the following two definitions as the special cases of path-level link penalty equilibrium (Definition 4).

Definition 5. An equilibrium flow is od-level link penalty equilibrium, if for each OD pair, all users are unanimous in considering the same penalty for each link of the network:

$$
\lambda_{a}^{k}=\lambda_{a}^{r s} \quad \forall a \in A^{k}, \quad k \in K_{r s}, r s .
$$

Definition 6. An equilibrium flow is link-level link penalty equilibrium, if all users are unanimous in considering the same penalty for each link of the network:

$$
\lambda_{a}^{k}=\lambda_{a} \quad \forall a \in A^{k}, \quad k \in K_{r s}, r s .
$$

The required condition for a link-level link penalty equilibrium $\left(\lambda_{a}^{k}=\lambda_{a}\right)$ is in fact equivalent to the condition imposed in Remark 3 . Hence, under this equilibrium, Condition (11-3) of the extended complementarity model is redundant, and the equilibrium model is equivalent to the Kuhn-Tucker conditions of BMW mathematical program with link capacity constraints:

$$
\begin{array}{cl}
\min & \sum_{a \in A} \int_{0}^{x_{a}} t_{a}(w) d w \\
\sum_{k \in K_{r s}} f_{k}=q_{r s} & \forall r s, \\
x_{a}=\sum_{r s} \sum_{k \in K_{r s}} \delta_{a k} f_{k} & \forall a \in A, \\
x_{a} \leq c_{a} & \forall a \in A, \\
f_{k} \geq 0 & \forall k \in K_{r s}, r s .
\end{array}
$$

In BMW optimization model, $\lambda_{a} \forall a \in A$ (as shown in Remark 3) represents the dual variable associated with the capacity constraint of link $a$. In Model (22)(26), the objective function is the well-known BMW maximization objective, and Constraints (23)-(26) represent the set of feasible flows.

\section{Numerical experiments}

Two numerical examples are presented in this section. The first example is designed to show the role of Condition (11-3) in defining the equilibrium. The complementarity model without Condition (11-3) represents the entire set of equilibria, whereas inclusion of Condition (11-3) restricts the solution to the set of NE. The second example is designed to show the set of equilibrium flows based on different link penalty concepts.

Example 1. Figure 3 presents a simple example which is used in Marcotte et al. [6]. Tables 1 and 2 provide the details of the links and paths.

Marcotte et al. [6] show that for $3 \leq t \leq 5$, the flows of Table 2 are feasible, and they comply with Definition 1, because the only path with remaining capacity is path 3 , and the travel time of paths 1 and 2 (which have positive flows) is smaller than the travel time of path 3 . They also show that the solution is more "natural" when $t=3$, because when $t>3$, a total of $t-3$ travelers can divert from path 2 to path 1 to reduce their travel time.

Also according to Lemma 3 or Condition (8), a feasible flow is the equilibrium flow when $t=3$, since for $t>3$ we have:

$$
\Delta_{21}(f)=\min \left\{f_{2}, c_{1}-x_{1}\right\}=\min \{t, t-3\}>0,
$$

which contradicts Lemma 3 , since $175=T_{2}>T_{1}=$

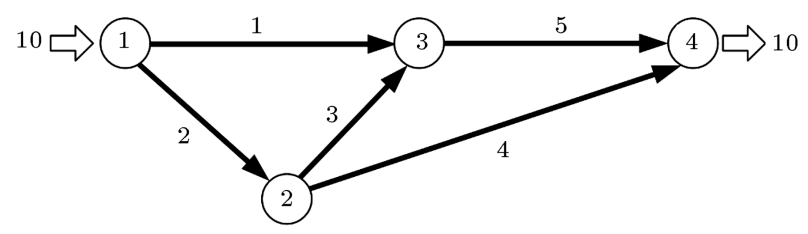

Figure 3. Network of Example 1.

Table 1. Details of the links of Example 1.

\begin{tabular}{ccccc}
\hline Link & $\boldsymbol{c}_{\boldsymbol{a}}$ & $\boldsymbol{t}_{\boldsymbol{a}}$ & $\boldsymbol{x}_{\boldsymbol{a}}$ & $\boldsymbol{c}_{\boldsymbol{a}}-\boldsymbol{x}_{\boldsymbol{a}}$ \\
\hline 1 & 2 & 50 & $5-t$ & $t-3$ \\
2 & $\infty$ & 50 & $5+t$ & $\infty$ \\
3 & $\infty$ & 75 & $t$ & $\infty$ \\
4 & $\infty$ & 150 & 5 & $\infty$ \\
5 & 5 & 50 & 5 & 0 \\
\hline
\end{tabular}

Table 2. Details of the paths of Example 1.

\begin{tabular}{ccccc}
\hline Path $\boldsymbol{k}$ & $\begin{array}{c}\text { Links of } \\
\text { path } \boldsymbol{k}\end{array}$ & $\boldsymbol{T}_{\boldsymbol{k}}$ & $\boldsymbol{f}_{\boldsymbol{k}}$ & $\begin{array}{c}\text { Remaining } \\
\text { capacity }\end{array}$ \\
\hline 1 & 1,5 & 100 & $5-t$ & 0 \\
2 & $2,3,5$ & 175 & $t$ & 0 \\
3 & 2,4 & 200 & 5 & $\infty$ \\
\hline
\end{tabular}


100. For $t=3$, the solution is in equilibrium, because $\Delta_{21}=\Delta_{31}=\Delta_{32}=0, \Delta_{12}=2, \Delta_{13}=2$, and $\Delta_{23}=$ 3 , which satisfies Lemma 3 (i.e. $T_{k} \leq T_{l}$ ).

Model (11) without Condition (11-3) is presented for Example 1 as follows:

$$
\begin{aligned}
& \left(100+\lambda_{1}^{1}+\lambda_{5}^{1}-u\right) f_{1}=0 \\
& \left(175+\lambda_{5}^{2}-u\right) f_{2}=0 \\
& (200-u) f_{3}=0 \\
& f_{1}+f_{2}+f_{3}=10 \\
& \left(2-f_{1}\right) \lambda_{1}^{1}=0 \\
& \left(5-f_{1}-f_{2}\right) \lambda_{5}^{1}=0 \\
& \left(5-f_{1}-f_{2}\right) \lambda_{5}^{2}=0 \\
& 2-f_{1} \geq 0 \\
& 5-f_{1}-f_{2} \geq 0 .
\end{aligned}
$$

It is easy to see that for $3<t \leq 5$, the flows $f_{1}=$ $5-t, f_{2}=t$, and $f_{3}=5$ satisfy Model (11) without Condition (11-3), because it is sufficient to use:

$$
u=200, \quad \lambda_{1}^{1}=0, \quad \lambda_{5}^{1}=100, \quad \lambda_{5}^{2}=25 .
$$

However, the above solution does not satisfy Condition (11-3), because for $i=1, j=3, k=2$, and $l=1$, Condition (11-3) becomes:

$$
(125-50)\left(2-f_{1}\right) f_{2} \leq 0,
$$

which cannot be true, since $f_{1}<2$ and $f_{2}>0$. However, for $t=3$, the flows $f_{1}=2, f_{2}=3$, and $f_{3}=5$ satisfy all the constraints of Model (11), because it is sufficient to use:

$$
u=200, \quad \lambda_{1}^{1}+\lambda_{5}^{1}=100, \quad \lambda_{5}^{2}=25 .
$$

Moreover, this solution is also the solution of BMW mathematical program, because it is sufficient to use:

$$
\lambda_{1}^{1}=75, \quad \lambda_{5}^{1}=\lambda_{5}^{2}=25 .
$$

\begin{tabular}{|c|c|c|c|c|c|}
\hline $\begin{array}{c}\text { Link } \\
\quad a\end{array}$ & $c_{a}$ & $t_{a}\left(x_{a}\right)$ & $\begin{array}{c}\text { Link } \\
\quad a\end{array}$ & $c_{a}$ & $t_{a}\left(x_{a}\right)$ \\
\hline 1 & $\infty$ & 1 & 5 & $\infty$ & 0 \\
\hline 2 & 1 & $x_{2} / 2$ & 6 & $\infty$ & 5 \\
\hline 3 & 1 & $x_{3} / 2$ & 7 & $\infty$ & $x_{7}$ \\
\hline 4 & $\infty$ & 1 & 8 & $\infty$ & 0 \\
\hline
\end{tabular}

To sum up, Definition 1 is equivalent to Model (11) without Constraint (11-3), whereas inclusion of Constraints (11-3) leads to NE.

Example 2. The following example, as illustrated in Figure 4, is a combination of two simple examples from Correa et al. [11]. The network includes one origin node, two destination nodes, and a total of 5 nodes and 8 links. The details of the links and paths are provided in Tables 3 and 4, respectively.

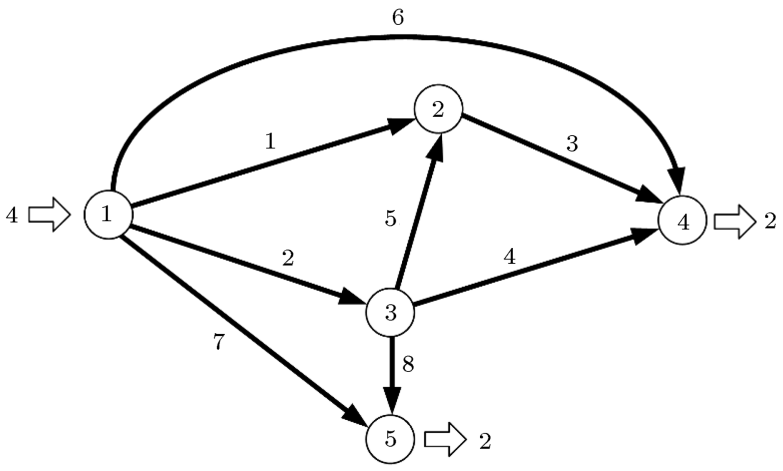

Figure 4. Network of Example 2.

Table 3. Details of the links of Example 2.

Table 4. Details of the paths of Example 2.

\begin{tabular}{ccccc}
\hline $\begin{array}{c}\text { Path } \\
\boldsymbol{k}\end{array}$ & $\boldsymbol{r}$ - $\boldsymbol{s}$ & $\begin{array}{c}\text { Links } \\
\text { of path } \\
\boldsymbol{k}\end{array}$ & $\begin{array}{c}\text { Flow } \\
\text { of path } \\
\boldsymbol{k}\end{array}$ & $\begin{array}{c}\text { Link flow } \\
\text { based on } \\
\text { path flows }\end{array}$ \\
\hline 1 & $1-4$ & 1,3 & $f_{1}$ & $x_{1}=f_{1}$ \\
2 & $1-4$ & 2,4 & $f_{2}$ & $x_{2}=f_{2}+f_{3}+f_{6}$ \\
3 & $1-4$ & $2,5,3$ & $f_{3}$ & $x_{3}=f_{1}+f_{3}$ \\
4 & $1-4$ & 6 & $f_{4}$ & $x_{4}=f_{2}, x_{5}=f_{3}$ \\
5 & $1-5$ & 7 & $f_{5}$ & $x_{6}=f_{4}, x_{7}=f_{5}$ \\
6 & $1-5$ & 2,8 & $f_{6}$ & $x_{8}=f_{6}$ \\
\hline
\end{tabular}

According to Eq. (7), flow $f$ is feasible, if the following constraints are satisfied.

$$
\begin{aligned}
& f_{1}+f_{2}+f_{3}+f_{4}=2, \\
& f_{5}+f_{6}=2, \\
& f_{2}+f_{3}+f_{6} \leq 1, \\
& f_{1}+f_{3} \leq 1 \\
& f_{k} \geq 0 \quad k=1, \cdots, 6 .
\end{aligned}
$$

A feasible solution can be obtained for all $f_{4}$ and $f_{6}$ that satisfy the following conditions:

$$
0 \leq f_{4} \leq 2, \quad 0 \leq f_{6} \leq 1, \quad f_{6} \leq f_{4} .
$$

Note that the right-most relation in Eq. (28) results by subtracting Eq. (27-1) from the sum of Eq. (27-3) and (27-4) to get $f_{4} \geq f_{6}+f_{3}$, and also note that $f_{3} \geq 0$. 


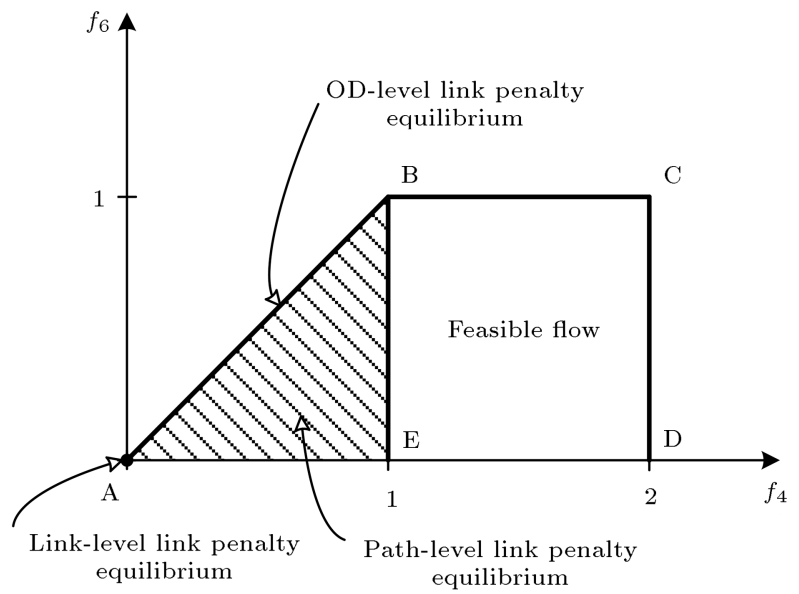

Figure 5. Equilibrium flows of Example 2.

The feasible flow based on $f_{4}$ and $f_{6}$ is the polygon ABCDE in Figure 5. We present the set of feasible flows that are in equilibrium, and identify the equilibria.

Path-level link penalty equilibrium: The triangle ABE in Figure 5 presents all feasible flows (where links 2 and 3 are saturated) that are the solution to the extended complementarity model. These are the flows that satisfy the following:

$$
\begin{aligned}
& 0 \leq f_{6} \leq f_{4} \leq 1, \quad f_{2}+f_{3}+f_{6}=1, \\
& f_{1}+f_{3}=1 .
\end{aligned}
$$

In this example, any flow that does not saturate links 2 and 3 cannot lead to the path-level link penalty equilibrium;

Od-level link penalty equilibrium: All flows that fall on line $\mathrm{AB}$ of Figure 5 (where links 2 and 3 are saturated) are the solutions to the extended complementarity model with the condition $\lambda_{a}^{k}=\lambda_{a}^{r s}$. That is, all flows that satisfy the following conditions are od-level link penalty equilibria:

$$
\begin{aligned}
& 0 \leq f_{6}=f_{4} \leq 1, \quad f_{2}+f_{3}+f_{6}=1, \\
& f_{1}+f_{3}=1 .
\end{aligned}
$$

Link-level link penalty equilibrium: The flow $f_{6}=f_{4}=$ 0 (point A in Figure 5) is the only link-level link penalty equilibrium of the extended complementarity model with $\lambda_{a}^{k}=\lambda_{a}$.

The results of Example 2 are summarized in Table 5, and the total system travel time (i.e., $\sum_{a} x_{a} t_{a}$ ) is provided, as well, in the last row of the table. It is interesting to note that the total system travel time for the link-level link penalty equilibrium flow is the minimum among all equilibrium flows.
Table 5. Equilibrium flows under the different link penalty concepts of Example 2.

\begin{tabular}{cccc}
\hline $\begin{array}{c}\text { Path } \\
\boldsymbol{k}\end{array}$ & $\begin{array}{c}\text { Path-level } \\
\text { link penalty } \\
\mathbf{0} \leq \boldsymbol{f}_{\mathbf{6}} \leq \boldsymbol{f}_{\mathbf{4}} \leq \mathbf{1}\end{array}$ & $\begin{array}{c}\text { OD-level } \\
\text { link penalty } \\
\mathbf{0} \leq \boldsymbol{f}_{\mathbf{4}} \leq \mathbf{1}\end{array}$ & $\begin{array}{c}\text { Link-level } \\
\text { link } \\
\text { penalty }\end{array}$ \\
\hline 1 & $1-f_{4}+f_{6}$ & 1 & 1 \\
2 & $1-f_{4}$ & $1-f_{4}$ & 1 \\
3 & $f_{4}-f_{6}$ & 0 & 0 \\
4 & $f_{4}$ & $f_{4}$ & 0 \\
5 & $2-f_{6}$ & $2-f_{4}$ & 2 \\
6 & $f_{6}$ & $f_{4}$ & 0 \\
$\sum_{a} x_{a} t_{a}$ & $7+3 f_{4}-3 f_{6}+f_{6}^{2}$ & $7+f_{4}^{2}$ & 7 \\
\hline
\end{tabular}

\section{Summary and conclusions}

This paper presents a complementarity traffic equilibrium model with capacity constraints. These networks are gaining importance for reasons that the uncapacitated networks may produce unreal high user costs in order to deter flow from entering a congested link, and hence overestimating the total travel cost (time) in the network. Currently, the wealth of studies on uncapacitated networks has established the idea that User Equilibrium (UE) is equivalent to NE when volume-delay functions are separable, continuous, nondecreasing and non-negative. The same conclusion, however, does not apply in capacitated networks. One task in this article is to redefine wardrop's first principle for capacitated networks.

This paper investigates the connection between UE and NE by proposing a complementarity equilibrium model, extending Wardrop's first principle in capacitated networks. The problem is introduced by the help of a motivating example, and by showing that an existing definition of equilibrium in the literature for the capacitated networks does not strictly define NE and its relation with UE. The relations between UE and other equilibrium concepts such as NE are important in that they may be used as selection criteria to guide flow patterns toward the preferred one, which may help gaining a more efficient and fairer flow pattern in some cases. Thus, a second task in this article is to show the relations between some equilibrium concepts to open a new avenue to further research in this area, including the applicability of the existing analytical models for the uncapacitated UE flow problems to their capacitated counterparts.

The complementarity problem defined to describe NE in the capacitated network flow problem is such that its special cases represent subsets of equilibria. It can understand when an equilibrium is an NE. The paper then investigates the stability of the equilibrium 
and shows that under special conditions, the complementarity model provides the same solution as the BMW optimization model.

\section{Nomenclature}

$N \quad$ Set of network nodes

$A \quad$ Set of network links

$R \subseteq N \quad$ Set of origin nodes

$S \subseteq N \quad$ Set of destination nodes

$q_{r s}>0$ Travel demand from origin $r$ to destination $s$

$K_{r s} \quad$ Set of paths from origin $r$ to destination $s$

$\delta_{a k} \quad$ Link-path incidence index which is equal to 1 if link $a$ is in path $k$ and 0 otherwise

$f_{k} \quad$ Traffic flow of path $k$

$f \quad$ Vector of path flows

$x_{a} \quad$ Traffic flow of link $a$

$c_{a} \quad$ Capacity of link $a$

$x \quad$ Vector of link flows

$t_{a}\left(x_{a}\right) \quad$ Volume-delay function of link $a$ which is continuous, non-negative, and non-decreasing

$A^{k} \quad=\left\{a \in A \mid \delta_{a k}=1\right\}$

$\varphi_{k l}(f) \quad$ The maximum flow that can be transferred from path $k$ to path $l$

$K_{r s}^{i j} \quad=\left\{k \in K_{r s} \mid \exists\right.$ a sub-path in $k$ that connects node $i$ to node $j\}$

$K_{r s}^{1} \quad=\left\{k \in K_{r s} \mid f_{k}>0, \bar{c}_{k}>0\right\}$; part of the set $K_{r s}$

$K_{r s}^{2}=\left\{k \in K_{r s} \mid f_{k}>0, \bar{c}_{k}=0\right\}$; part of the set $K_{r s}$

$K_{r s}^{3} \quad=\left\{k \in K_{r s} \mid f_{k}=0, \bar{c}_{k}=0\right\}$; part of the set $K_{r s}$

$K_{r s}^{4} \quad=\left\{k \in K_{r s} \mid f_{k}=0, \bar{c}_{k}>0\right\}$; part of the set $K_{r s}$

$\delta_{a k}^{i j} \quad 1$, if $a$ is in a sub-path of $k$ that connects node $i$ to node $j$; 0 otherwise

$\bar{c}_{a} \quad$ The remaining capacity of link $a=c_{a}-x_{a}$

$\bar{c}_{k} \quad$ The remaining capacity of path $k=\min _{a \in A^{k}} \bar{c}_{a}$

$\bar{c}_{k}^{i j} \quad$ The remaining capacity of part of path $k$ that connects $i$ to $j=\min _{a \in A: \delta_{a k}^{i j}=1}\left\{c_{a}-x_{a}\right\}$

$T_{k}^{i j} \quad$ The travel time of path $k$ from node $i$ to node $j=\sum_{a \in A} \delta_{a k}^{i j} t_{a}(x)$ $\lambda_{a}^{k} \quad$ The penalty of using saturated link $a$ (for which $x_{a}=c_{a}$ ) in path $k$

$\beta_{k} \quad=\sum_{a \in A} \delta_{a k} \lambda_{a}^{k}$

$\beta_{k}^{i j} \quad=\sum_{a \in A} \delta_{a k}^{i j} \lambda_{a}^{k}$

$k^{i j} \quad$ Part of path $k$ that extends from node $i$ to node $j$

\section{References}

1. Morlok, E.K., Introduction to Transportation Engineering and Planning, McGraw-Hill College, New York (1978).

2. Beckmann, M., McGuire, C., and Winsten, C., Studies in the Economics of Transportation, Yale University Press, New Haven, Conn (1956).

3. Zhang, C., Chen, X., and Sumalee, A. "Robust Wardrop's user equilibrium assignment under stochastic demand and supply: expected residual minimization approach", Transportation Research Part B: Methodological, 45(3), pp. 534-552 (2011).

4. Nie, Y.M. "Equilibrium analysis of macroscopic traffic oscillations", Transportation Research Part B: Methodological, 44(1), pp. 62-72 (2010).

5. He, F., Yin, Y., Shirmohammadi, N., and Nie, Y.M. "Tradable credit schemes on networks with mixed equilibrium behaviors", Transportation Research Part B: Methodological, 57, pp. 47-65 (2013).

6. Marcotte, P., Nguyen, S., and Schoeb, A. "A strategic flow model of traffic assignment in static capacitated networks", Operations Research, 52(2), pp. 191-212 (2004).

7. Patriksson, M. "The traffic assignment problem: models and methods", Utrecht, VSP, BV, The Netherlands (1994).

8. Branston, D. "Link capacity functions: A review", Transportation Research, 10(4), pp. 223-236 (1976).

9. Larsson, T. and Patriksson, M. "An augmented Lagrangean dual algorithm for link capacity side constrained traffic assignment problems", Transportation Research Part B: Methodological, 29(6), pp. 433-455 (1995).

10. Larsson, T. and Patriksson, M. "Side constrained traffic equilibrium models-analysis, computation and applications", Transportation Research Part B: Methodological, 33(4), pp. 233-264 (1999).

11. Correa, J.R., Schulz, A.S., and Stier-Moses, N.E. "Selfish routing in capacitated networks", Mathematics of Operations Research, 29(4), pp. 961-976 (2004).

12. Zhang, D., Nagurney, A., and Wu, J. "On the equivalence between stationary link flow patterns and traffic network equilibria", Transportation Research Part B: Methodological, 35(8), pp. 731-748 (2001).

13. Yang, F. and Zhang, D. "Day-to-day stationary link flow pattern", Transportation Research Part B: Methodological, 43(1), pp. 119-126 (2009). 
14. Smith, M.J. "The stability of a dynamic model of traffic assignment-an application of a method of Lyapunov", Transportation Science, 18(3), pp. 245-252 (1984).

15. Jin, W.-L. "On the stability of user equilibria in static transportation networks", Transportmetrica, 4(1), pp. $1-17$ (2008).

16. Jin, W.-L. "A dynamical system model of the traffic assignment problem", Transportation Res earch Part B: Methodological, 41(1), pp. 32-48 (2007).

17. Szeto, W. and Lo, H.K. "Dynamic traffic assignment: properties and extensions", Transportmetrica, 2(1), pp. 31-52 (2006).

18. Nasri, M. and Sosa, W. "Equilibrium problems and generalized Nash games", Optimization, 60(8-9), pp. 1161-1170 (2011).

19. Rosenthal, R.W. "The network equilibrium problem in integers", Networks, 3(1), pp. 53-59 (1973).

20. Devarajan, S. "A note of network equilibrium and noncooperative games", Transportation Research Part B: Methodological, 15(6), pp. 421-426 (1981).

21. Levinson, D. "Micro-foundations of congestion and pricing: A game theory perspective", Transportation Research Part A: Policy and Practice, 39(7-9), pp. 691-704 (2005).

22. Zou, X. and Levinson, D. "A multi-agent congestion and pricing model", Transportmetrica, 2(3), pp. 237249 (2006).

23. Han, Q., Dellaert, B., Van Raaij, F., and Timmermans, H. "Modelling strategic behaviour in anticipation of congestion", Transportmetrica, 3(2), pp. 119-138 (2007).

24. Anshelevich, E., Dasgupta, A., Kleinberg, J., Tardos, E., Wexler, T., and Roughgarden, T. "The price of stability for network design with fair cost allocation", SIAM Journal on Computing, 38(4), pp. 1602-1623 (2008).

25. Li, C., Gopalarao, S., and Ray, T. "A path-based flow formulation for the traffic assignment problem", Transportation Planning and Technology, 39(6), pp. 597-611 (2016).

26. Schulz, A.S. and Stier Moses, N. "On the performance of user equilibria in traffic networks", Working Paper 4274-02, MIT Sloan School of Management (2003).

27. Jahn, O., Möhring, R.H., Schulz, A.S., and StierMoses, N.E. "System-optimal routing of traffic flows with user constraints in networks with congestion", Operations Research, 53(4), pp. 600-616 (2005).

28. Poorzahedy, H. and Bushehri, S.N.S. "Pessimistic equilibrium flow and its network design implications", European Journal of Transport and Infrastructure Research, 6(2), pp. 113-130 (2006).
29. Tobin, R.L. and Friesz, T.L. "Sensitivity analysis for equilibrium network flow", Transportation Science, 22(4), pp. 242-250 (1988).

30. Daganzo, C.F. "On the traffic assignment problem with flow dependent costs-II", Transportation Research, 11(6), pp. 439-441 (1977).

31. Daganzo, C.F. "On the traffic assignment problem with flow dependent costs-I", Transportation Research, 11(6), pp. 433-437 (1977).

32. Boyce, D., Janson, B., and Eash, R. "The effect on equilibrium trip assignment of different link congestion functions", Transportation Research Part A: General, 15(3), pp. 223-232 (1981).

33. Nie, Y., Zhang, H., and Lee, D.-H. "Models and algorithms for the traffic assignment problem with link capacity constraints", Transportation Research Part B: Methodological, 38(4), pp. 285-312 (2004).

34. Hearn, D. "Bounding flows in traffic assignment models", in Research Report 80-4, Department of Industrial and Systems Engineering, University of Florida Gainesville, FL (1980).

35. Daganzo, C.F. "Queue spillovers in transportation networks with a route choice", Transportation Science, 32(1), pp. 3-11 (1998).

36. Hearn, D. and Ribera, J. "Bounded flow equilibrium problems by penalty methods", In Proceedings of IEEE International Conference on Circuits and Computers, Institute of Electrical and Electronics Engineers, New York (1980).

37. Shahpar, A.H., Aashtiani, H.Z., and Babazadeh, A. "Dynamic penalty function method for the side constrained traffic assignment problem", Applied Mathematics and Computation, 206(1), pp. 332-345 (2008).

38. Yang, H. and Yagar, S. "Traffic assignment and traffic control in general freeway-arterial corridor systems", Transportation Research Part B: Methodological, 28(6), pp. 463-486 (1994).

39. Yang, H. and Yagar, S. "Traffic assignment and signal control in saturated road networks", Transportation Research Part A: Policy and Practice, 29(2), pp. 125139 (1995).

40. Haurie, A. and Marcotte, P. "On the relationship between Nash-Cournot and Wardrop equilibria", Networks, 15(3), pp. 295-308 (1985).

41. Roughgarden, T. and Tardos, $\dot{\mathrm{E}}$. "How bad is selfish routing?", Journal of the ACM (JACM), 49(2), pp. 236-259 (2002).

42. Wardrop, J.G. "Some theoretical aspects of road traffic research", OR: Operational Research Quarterly, 4(4), pp. $72-73$ (1952).

43. Aashtiani, H.Z. "The multi-modal traffic assignment problem", PhD Dissertation, Massachusetts Institute of Technology, Boston, MA, USA (1979). 


\section{Biographies}

Hedayat Zokaei Aashtiani is Professor of Transportation at Sharif University of Technology, Department of Civil Engineering. He received his $\mathrm{PhD}$ in Operations Research from MIT in 1979. His research interests include mathematical programming, integer programming, network flow, traffic equilibrium, traffic assignment, and network design. He has been involved in several comprehensive urban transportation studies for large cities in Iran.

Hossain Poorzahedy is Professor of Transportation at Sharif University of Technology, Department of Civil Engineering. He received his $\mathrm{PhD}$ from Northwestern University (1980), MS from Stanford University (1976), and BS from Shiraz University (1975). His research interests are in various aspects of transportation network design: solution methods; large real network case studies; network reliability, capacity, sustainability, resiliency, and chaotic behavior; network pricing; and network development finance through Build-Operate-
Transfer schemes. His recent works bring together land use and transportation, transit-oriented development, air pollution and disaster management as related to transportation.

Mehdi Nourinejad received his PhD degree specializing in Operations Research and Transportation. He is currently a limited term faculty at the Rotman School of Management and is an Adjunct Professor at the Ted Rogers School of Management. Dr Nourinejad's research focuses on optimal planning and control of sustainable transportation systems in smart cities with autonomous vehicles. He has successfully published his research in peer-reviewed journals. $\mathrm{He}$ is on the editorial board of "Transportation Research Part E: Logistics and Transportation Review," a journal in the Transportation field. His research on autonomous vehicles has received media coverage and is published in outlets including Forbes, University of Toronto Engineering news, Popular Science, Design Quarterly, Global News, Gizmag, Science Daily, and Tech Xplore. 